\title{
A design concept of active cooling for tailored forming workpieces during induction heating
}

\author{
Caner-Veli Ince ${ }^{1} \cdot$ Anna Chugreeva ${ }^{2} \cdot$ Christoph Böhm $^{3} \cdot$ Fadi Aldakheel $^{3} \cdot$ Johanna Uhe $^{2} \cdot$ Peter Wriggers $^{3}$. \\ Bernd-Arno Behrens ${ }^{2} \cdot$ Annika Raatz ${ }^{1}$
}

Received: 14 August 2020 / Accepted: 31 January 2021 / Published online: 26 February 2021

(c) The Author(s) 2021

\begin{abstract}
The demand for lightweight construction is constantly increasing. One approach to meet this challenge is the development of hybrid components made of dissimilar materials. The use of the hybrid construction method for bulk components has a high potential for weight reduction and increased functionality. However, forming workpieces consisting of dissimilar materials requires specific temperature profiles for achieving sufficient formability. This paper deals with the development of a specific heating and cooling strategy to generate an inhomogeneous temperature distribution in hybrid workpieces. Firstly, the heating process boundaries with regard to temperature parameters required for a successful forming are experimentally defined. Secondly, a design based on the obtained cooling strategy is developed. Next a modelling embedded within an electro-thermal framework provides the basis for a numerical determination of admissible cooling rates to fulfil the temperature constraint. Here, the authors illustrate an algorithmic approach for the optimisation of cooling parameters towards an effective minimum, required for applicable forming processes of tailored forming.
\end{abstract}

Keywords Tailored forming $\cdot$ Active cooling $\cdot$ Numerical simulation $\cdot$ Electro-thermal coupling

\section{Introduction}

The continuous increase of lightweight trends aiming at the reduction of fuel consumption and $\mathrm{CO}_{2}$ emissions leads to increased requirements on technical components [1]. Since mono-materials, commonly used for conventional components, are not able to fulfil the conflicting needs such as enhanced performance, low weight and compact design at the same time, development of advanced manufacturing strategies or processes is necessary [2]. By facing the need for locally adapted material response, hybrid constructions combining the benefits of different materials in a single component have shown promising results in early research.

Caner-Veli Ince

ince@match.uni-hannover.de

1 Leibniz Universität Hannover, Institute of Assembly Technology, An der Universität 2, Garbsen 30823, Germany

2 Leibniz Universität Hannover, Institute of Forming Technology and Machines, An der Universität 2, Garbsen 30823, Germany

3 Leibniz Universität Hannover, Institute of Continuum Mechanics, An der Universität 1, Garbsen 30823, Germany
When addressing complex geometric properties of the joining zone, well-established production process chains with the classical approach of near-net shape forming followed by joining might fail. An alternative method, where joining and forming is carried out in a single process step, allows for the production of complex shapes. However, it is challenging to ensure uniform bonding quality due to local inhomogeneous material flow and differences in the plastic strain [3]. In contrast, the novel technology of tailored forming, investigated by CRC 1153, leads to relevant results by first joining and subsequent forming [4]. Here, the initial compounds are also maintained or improved during forming. Production of workpieces is carried out by, for example, deposition or friction welding as well as by co-extrusion, where joining of the reinforcing element and the matrix material is performed using pressure welding. Forming to the final geometry is implemented by such processes as hot forging, impact extrusion or cross wedge rolling. The process chain is finalised by machining and heat treatment in order to set the operational properties to the hybrid components. Adjusting the process route according to the required geometrical and mechanical properties, complex geometric structures 
of hybrid material type are capable to be treated with the tailored forming technology.

Regarding dissimilar material combinations, temperature distribution before forming is one of the most demanding tasks in tailored forming. Forging complex geometric structures requires sufficient formability, which can be reached at elevated forming temperatures [5]. In hybrid workpieces combining dissimilar materials, this is rather challenging due to different material-specific properties (e.g. flow stress, formability, melting temperature etc.). Ideal contact properties of the compound result in a narrow processing window and limitations for the heat distribution in the hybrid workpiece.

This paper deals with the development of an advanced heating strategy for steel-aluminium workpieces to reach the required heat distribution. The unique feature of the new concept is the cooling of the workpieces during the induction heating. With this solution, a delay of aluminium melting is expected, thus achieving a higher steel temperature at the same time. In addition to better formability, higher forming temperature can be advantageous for the microstructure, which affects the resulting mechanical properties. A constitutive framework has to address the heat transfer, acting on the macroscopic length scale. Thermal effects in material modelling are widely investigated in literature, e.g. gradient-extended thermo-plasticity effects in [6] and in [7], modelling within microscopic length scales in [8], virtual element method (VEM) for thermo-mechanics in [9]. Coupled electric formulations can be found with regards to an incremental framework for ferroelectricity in [10], by including computational homogenization of microstructural effects in [11] and with addressing fracture in [12]. This paper describes the main milestones in the development and numerical design of the cooling process, whereby experimentally measured temperature profiles provide the foundation.

\section{Survey of the current literature}

This chapter provides an overview of heating techniques to reach specific heat distribution in objects. Afterwards the main aspects of cooling processes are shown.

\subsection{Tailored heating}

In the context of inhomogeneous temperature distribution, induction heating represents an interesting approach. Here, heat is induced by eddy currents, which occur in the alternating electromagnetic field. The induction coil can be positioned locally, if the heating is required in certain regions of the workpieces to achieve an axial temperature gradient. Guo et al. investigated the bending process of a steel pipe, where induction heating was applied directly in the deformation zone [13]. Song and Moon used this principle for local heating of a workpiece section for the forging of marine crankshafts [14]. Goldstein et al. studied the induction heating of serially arranged hybrid workpieces made of steel and aluminium [15]. The coil was locally placed around the steel part, where the heat was induced. The aluminium temperature was increased by heat conduction from the steel part. In order to achieve a radial temperature difference in coaxially arranged hybrid workpieces, the skin-effect, which occurs at high operating frequencies during the induction heating, can be used [16]. The eddy currents are more concentrated at the surface close to the induction coil. Using the example of steel-aluminium workpieces, it is possible to intensively heat only the steel part, while the aluminium temperature increases due to the heat dissipation from the steel. Kosch and Behrens used the simultaneous induction heating of steel and aluminium cylinders assembled with a little gap before compound forging [17]. The concept was conducted with an outer induction coil. Cavdar et al. investigated hot hydroforging processes of aluminium billets encapsulated in steel sheath [18]. The bi-metal preforms (without metallurgical bonding) were heated up from the outside to a temperature up to $1200{ }^{\circ} \mathrm{C}$, whereas the aluminium core was formed in the molten state.

\subsection{Cooling process}

After a temperature gradient has been generated, it is necessary to maintain or even increase this gradient by the use of a cooling process. The industry uses cooling processes to extract much energy from the material in a short time. This way, the structural and mechanical properties of the material can be manipulated. Depending on the cooling rates and starting temperatures, different heat treatment methods like quenching or tempering are realised. For this purpose, the hot object is brought into contact with a fluid that has a significantly lower temperature. This imbalance creates a temperature gradient which is counteracted by a heat flow $\dot{q}$. In order of $\dot{q}$ the temperature of the fluid increases. If the boiling temperature of the fluid is exceeded, a phase transition occurs, which results in complicated fluiddynamic and thermodynamic phenomena that interact with each other. These have been investigated and described by Mayinger et al. [19]. In this context, the Leidenfrost effect must be taken into account $[20,21]$. This describes the phenomenon that a vapour film can form on the surface of the object, which completely separates the surface from the liquid, so called film boiling, and has an insulating effect. In Fig. 1 the Leidenfrost effect is illustrated. The figure shows a surface having a temperature above the boiling point of the surrounding fluid. The vapour film that forms has the thickness $\delta$ and the corresponding thermal conductivity coefficient $\lambda$, 


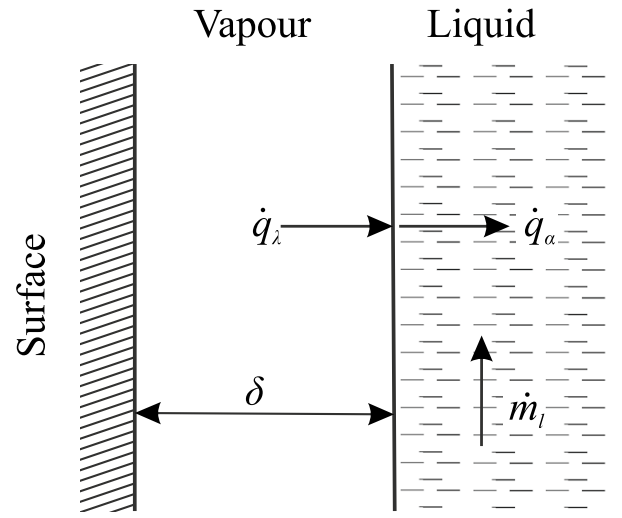

Fig. 1 Schematic illustration of the isolating vapour layer between surface and liquid at temperatures above Leidenfrost temperature

which results in a heat flow $\dot{q}_{\lambda}$. In the liquid medium, heat conduction takes place with a different coefficient, for which a heat flow in dependence of $\alpha$ is entered as $\dot{q}_{\alpha}$. Each phase has a specific range for their coefficients, shown in Table 1 , which explains the insulating effect of the vapour. The heat flow into the vapour and fluid creates a free convection $\dot{m}$, appearing in both phases.

As soon as the temperature falls below the boiling temperature of the fluid, which is also called Leidenfrost temperature, the vapour film breaks down and the heat dissipation increases again. The Leidenfrost temperature and the associated variable heat dissipation depends on the respective cooling process. This effect is regarded as a problem in conventional quenching processes, since high cooling rates are necessary. Nevertheless, in order to realise the temperature gradient required for tailored forming, the Leidenfrost effect can be utilised to create locally different cooling rates.

\section{Materials and methods}

The presented work refers to the process chain of the hybrid bearing bushing representing a lightweight concept, see Fig. 2, right. The temperature problems mentioned above occur during its manufacture, which is the reason for an exemplary design of the heating strategy. Following is a brief overview of the production process and the current solution approaches.

Table 1 Coefficients for gases and liquids under normal conditions [22]

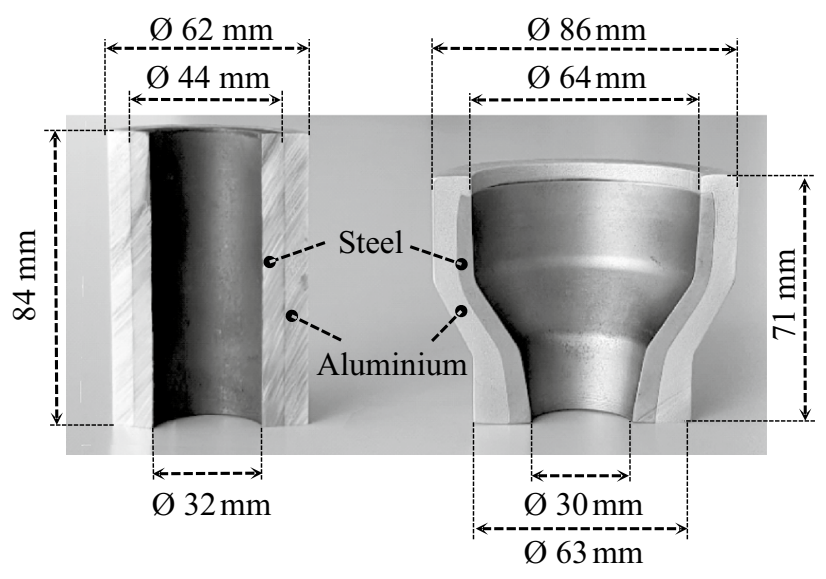

Fig. 2 Geometry of the hybrid workpiece (left) and of the bearing bushing (right)

\subsection{Bearing bushing}

The seat of the bearing balls is exposed to high rolling loads, therefore the inner surface requires the application of high performance and wear-resistant material. In this case, the steel is located on the internal diameter of the bearing bushing (20MnCr5). In other regions undergoing just structural load, lightweight material with high toughness such as aluminium can be used. In this case, an aluminium alloy (EN AW-6082) is located on the external diameter. The workpiece geometry is arranged in accordance to the load collective of the final part (Fig. 2, left). The required materials are joined to hollow steel-aluminium profiles by a co-extrusion process. After the joining, there is a metallurgical bond without intermetallic phase in the interface zone [23]. The presented work considers a strategy for setting the required temperature gradient in the above-described workpieces before the forging process.

\subsection{Induction heating}

In the case of the hybrid bearing bushing, a concept with an inner induction coil is used (Fig. 3). This arrangement allows to heat the steel part intensively on the internal diameter and, therefore, achieving a temperature gradient between steel and aluminium.

For sufficient forming without cracks, the steel temperature should be in a warm or hot forging temperature range, which begins above approx. $550{ }^{\circ} \mathrm{C}$ depending on the material [24]. The formability increases with the rising temperature. At the same time, the aluminium may not exceed a temperature of $500{ }^{\circ} \mathrm{C}$ to avoid any melting in the joining zone after the equalisation or forming in the semisolid state. The previous investigation on thermally pressed workpieces for the bearing bushing showed the feasibility of 


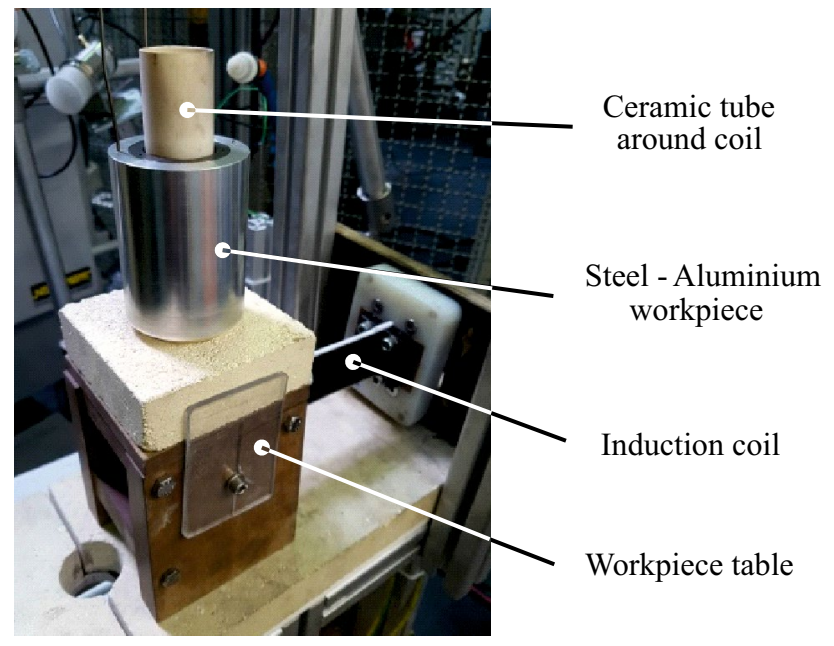

Fig. 3 Experimental setup of an induction heating for a coaxial hybrid specimen

the inhomogeneous heating strategy for the forging process [25]. However, the system had to be modified for utilising co-extruded profiles. This is because of the shorter temperature equalisation time due to better thermal contact in the joining zone. With the objective to shorten the transfer time to the forging tool, the induction heating unit was moved into the forging press. Therefore, the transfer time was reduced to $2.5 \mathrm{~s}$. The experimental heating tests were conducted with a medium frequency generator Huettinger TruHeat MF 3040 with a frequency range between 5 and $30 \mathrm{kHz}$. For achieving the largest possible temperature gradient between steel and aluminium within the shortest time, the maximum output power of $40 \mathrm{~kW}$ was pre-set. The maximum operating frequency during the heating was approx. $20.17 \mathrm{kHz}$. To define the process boundaries, the heating time was varied concerning the process boundaries described above. The temperature profiles were recorded on the reference points marked in Fig. 4 with steel sheathed thermocouples of type $\mathrm{K}$ with diameter of $1.5 \mathrm{~mm}$. The temperature measurements represented below in chapter 4 demonstrated that an improvement of the temperature gradient is required. Possible approaches are given in the next sections.

\subsection{Spray cooling}

After the introduction of the heating process the cooling follows. To maintain the processing temperatures of the hybrid workpieces, a cooling strategy is necessary. During the induction heating of the steel-aluminium workpieces, the heat conduction in the material causes the aluminium to heat up to such an extent that the melting temperature of the aluminium is exceeded. The consideration is to cool the aluminium side of the workpiece during induction heating.

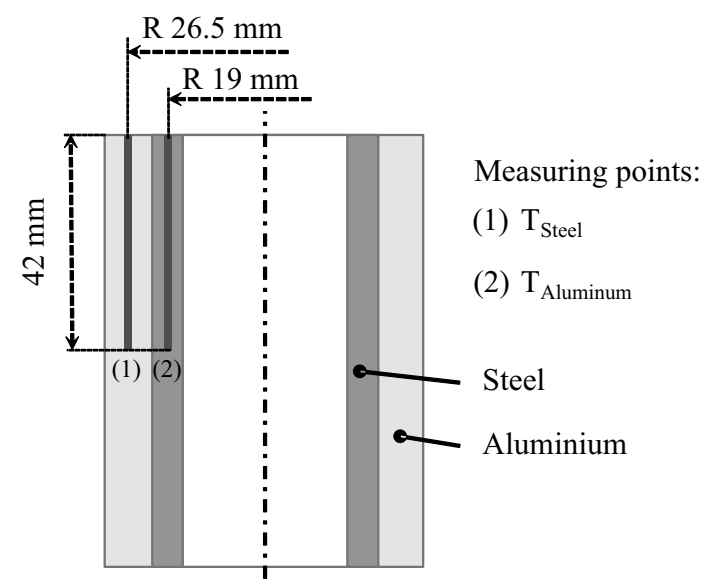

Fig. 4 Experimental measuring points for the induction heating tests

This enables a specific adjustment of heat distribution in the object.

In previous work, a concept of a cooling system was designed for this purpose and according to the boundary conditions at that time by Ince et al. [26]. Before modification of the heating process, as previously described, a longer transfer time (approx. $6 \mathrm{~s}$ ) was required. To maintain the temperature gradient, active cooling will be applied during the handling process from the induction coil to the forging press. The theoretically required cooling rates can be achieved by spray cooling. For this purpose a gripping system is equipped with nozzles, which cool the workpiece during heating and transport by using a water-air mixture. The designed gripping and spray cooling system is shown in Fig. 5. The system has variable shape grippers that are capable of handling hot forged parts. 8 nozzles are attached to the gripper, whereby the orientation of each can be adjusted individually. This ensures that the handled object is fully

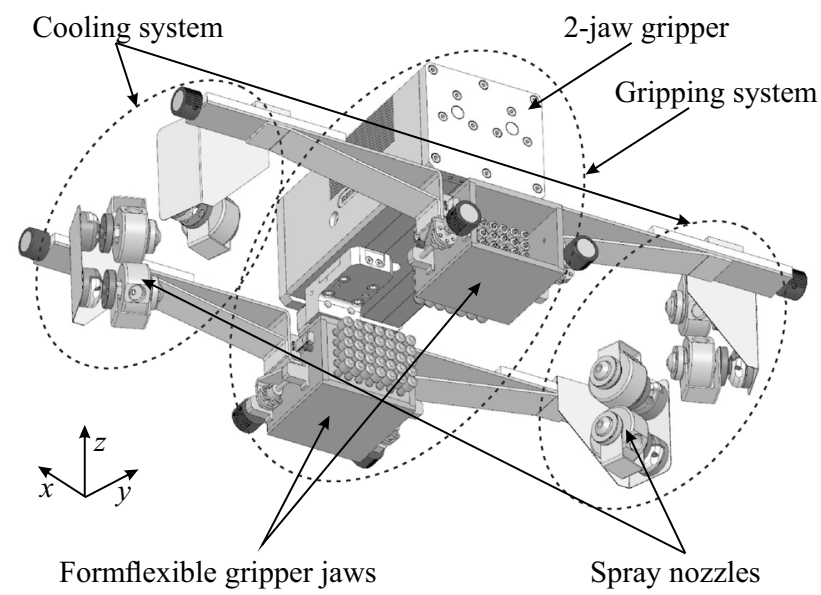

Fig. 5 Concept of gripping and cooling system for steel-aluminium hybrid components [26] 
covered by the medium, except the gripping points. To minimise the number of pins in contact and reduce the hidden surface the concept provides individually actuated pins. The medium is also distributed in the environment, since the transport is a dynamic process. Meanwhile, an optimisation of the heating and transport process of the bearing bushing has halved the transfer time. However, this means that the cooling strategy designed for aluminium-steel workpieces has to be improved according to the new conditions. Due to the shorter transfer time, it is only possible to cool during the heating phase. Now, a more comprehensive range of possible solutions can be investigated because stationary cooling processes that were previously not suitable for use are now possible.

\subsection{Immersion cooling}

In addition to the spray cooling, which can be used stationary, one of the suitable techniques for the changed conditions is immersion cooling. Thereby the object to be cooled is immersed in a bath and surrounded by the fluid. Jeschar et al. identified the temperature and its flow rate as the main parameters for the cooling rate [27]. A lower temperature and higher flow velocity maximise the heat flux density that can be extracted. Thus, high cooling rates are possible. The workpiece of the bearing bushing is surrounded by the fluid, which means that the steel side, which has to reach a much higher temperature than the aluminium, is also cooled. To minimise the heat dissipation from the steel, the Leidenfrost effect is utilised. The fluid must be selected depending on its Leidenfrost temperature, that film boiling starts only on the steel side. On the aluminium no film boiling is present, which is the reason why higher heat flux densities are achieved. Here the fluid used for cooling has an important role [28].

From the required heat distribution a heat flow results which must be dissipated. The exact determination of the amount of heat to be dissipated is important because the quality of the joining zone is directly dependent on it. Excessive heat dissipation would make forming of the partner material difficult or impossible because the temperature of the material would drop too far. This could damage the material or cause separation in the joining zone.

\subsection{Constitutive framework and computational approach}

For an efficient configuration of those temperature sensitive parameters, a numerical simulation enables a suitable heating and cooling strategy by simply testing different cases. Therefore, a model needs to consist of at least one thermal part, when assuming the heat through the induction source as constant. The modelling is embedded within a continuum based framework.

Let $\mathcal{B} \subset \mathbb{R}^{3}$ define the continuum and be of spatial dimension three. Moreover, $\mathcal{B}$ is parameterised by material points $\mathbf{x} \in \mathcal{B}$. Then, $\partial \mathcal{B} \subset \mathbb{R}^{2}$ denotes the surface of $\mathcal{B}$ with appropriate boundary conditions $\left\{\partial \mathcal{B}_{\mathcal{H}}, \partial \mathcal{B}_{T}\right\} \subset \partial \mathcal{B} \wedge \partial \mathcal{B}_{\mathcal{H}} \cap \partial \mathcal{B}_{T}=\emptyset$ of Dirichlet-type or Neumann-type, respectively, see Fig. 6 . The heating is specified by the process itself to be inductive and is yet assumed to be modelled with an effective term. This allows the consideration of a constant, time-independent term regarding the heating of $\mathcal{B}$. The cooling is also assumed to be constant and thus not sensitive to time. However, the heat conduction within the solid itself is time-sensitive. As a consequence, the constitutive framework of the problem shrinks to a pure thermal part. Whereas a heat source term models the electric and magnetic influence onto the temperature changes, is introduced later. Aspects for a coupled framework might be found in [29]. The governing equation for a transient heat transfer leads to

$\rho c \dot{T}+\operatorname{div}[\mathbf{q}]-\sum_{i} Q_{i}=0, \quad \mathbf{q}=-k \nabla T$.

Here, $\rho$ is the density of the solid, $\mathbf{q}$ denotes the heat flux of Fourier-Type [30], $Q_{i}$ is a particular source-term, associated to $i$-th type of source, $c$ is the specific heat capacity and $k$ is the thermal conductivity of the solid. The inductive heating, localised in the inner material of the hybrid specimen, is modelled through a source term, denoting the Joule-heating effect [31]

$Q_{\text {Joule }}=\mathbf{J} \cdot \mathbf{E}$,

where $\mathbf{J}$ denotes the current density and $\mathbf{E}$ is the electric field intensity vector. At this point, a thermo-electro-magnetic coupling enters the modelling, which is governed by the Maxwell-Equations, see [31]. The effect of an inductive heating source is modelled as an effective mean and is taken to be constant, which leads to a pure thermal consideration of the modelling. However, a constitutive relation between

Fig. 6 Continuum $\mathcal{B}$ with associated material point $\mathbf{x}$ and boundary conditions $\left\{\partial \mathcal{B}_{\mathcal{H}}, \partial \mathcal{B}_{T}\right\} \subset \partial \mathcal{B}$ applied to its surface $\partial \mathcal{B}$

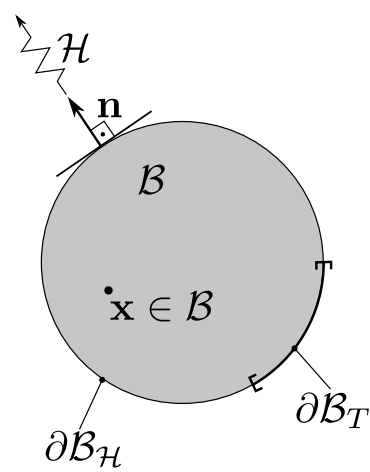


the current density and the electric field intensity is assumed to be of the form [32]

$\mathbf{J}=\sigma \mathbf{E}$,

where $\sigma$ is the electric conductivity and thus a material dependent property. This leads to the inductive heating source term by means of eddy currents yielding

$Q_{\text {Joule }}=\sigma|\mathbf{E}|^{2}$.

Within a thermal framework that accounts for heating due to an electric field, the skin effect controls the penetration depth of the inducted field into the material. The penetration depth can be accounted by following [33] with

$p_{d}=\sqrt{\frac{1}{\sigma \pi \mu f}}$,

with $f$ being the frequency of the current and $\mu$ being the permeability. The cooling, located on the outer surface of the specimen, is achieved through employment of a spraying or full immersion into a fluid. Such processes, when addressing thermal modelling frameworks, are convection dominant and exhibit a source term

$Q_{c}=h\left(T_{f l}-T\right)$.

Here, $h$ denotes the heat transfer coefficient and $T_{f l}$ is the temperature of the fluid, which is assumed to be a constant parameter. Thus, Eq. (1) is rewritten to

$\rho c_{v} \frac{\partial T}{\partial t}+\operatorname{div}[\mathbf{q}]-Q_{\text {Joule }}-Q_{c}=0$

Multiplication of Eq. (7) with a variation of the temperature $\delta T$ and integration over the domain $\mathcal{B}$ leads to the weak form [34]:

$$
\begin{array}{r}
\delta U(T)=\int_{\mathcal{B}} \delta T \rho c \frac{\partial T}{\partial t} \mathrm{~d} \Omega-\int_{\mathcal{B}}(\nabla \delta T)^{\mathrm{T}} \mathbf{q} \mathrm{d} \Omega \\
-\int_{\mathcal{B}} \delta T \sigma|\mathbf{E}|^{2} \mathrm{~d} \Omega-\int_{\partial \mathcal{B}} h\left(T_{f l}-T\right) \delta T \mathrm{~d} \Gamma=0 .
\end{array}
$$

Here, $\Omega$ denotes the volume of $\mathcal{B}$ and $\Gamma$ its surface. $\delta U(T)$ is the variation of a pseudo-potential, associated with the model problem. The heat flux is set to $\mathcal{H}=0$ on the boundary $\partial \mathcal{B}_{\mathcal{H}}$. The domain $\mathcal{B}$ is discretised by non-overlapping finite elements in $\mathbb{R}^{3}$, being either of tetrahedral or hexahedral shape. Applied shape functions are of polynomial order $p=2$ [35]. This scheme leads to a classical boundary value problem for the heat conduction within $\mathcal{B}$, which is solved in terms of a minimisation of a potential $U(T) \rightarrow \min$. The potential is assembled through all elements by application of the assembly operator, running over all elements $e$
$U(T)=\mathbf{A}_{U_{e}(T) .}$

The element residual $\mathbf{R}_{e}$ and the element tangent matrix $\mathbf{K}_{e}$ are then obtained through the employment of automated differentiation based formulation tools. This is achieved by utilising the symbolic mathematic software tool ACEGen [35] as a subpackage in the software MATHEMATICA, yields

$$
\begin{aligned}
& \mathbf{R}_{e}=\delta U_{e}(T)=\left.\frac{\partial U_{e}(T)}{\partial \mathbf{p}_{e}}\right|_{\left\{Q_{\text {Joule }}, Q_{c}\right\}=\text { const. }}, \\
& \mathbf{K}_{e}=\frac{\partial \mathbf{R}_{e}}{\partial \mathbf{p}_{e}} .
\end{aligned}
$$

Here, $\mathbf{p}_{e}=\operatorname{vec}\left\{T_{e}\right\}$ denotes the vector of unknowns at element level. The variation $\delta U_{e}(T)$ equals the derivation of the potential $U_{e}(T)$ with respect to the nodal degrees of freedom with consideration of constant source terms $\left\{Q_{\text {Joule }}, Q_{c}\right\}$ [35].

For obtaining a suitable parameter set $\left\{A_{c}, T_{f}, h\right\}$ for a successful cooling of the heated specimen, a minimisation scheme is employed. Furthermore, the algorithmic treatment enables the configuration of the set of cooling parameters with respect to certain aspects like cooling surface area $A_{c}$, fluid temperature $T_{f l}$ or at least the type of fluid itself, denoted by the heat transfer coefficient $h$. First, the maximum of the surface area, which is available for application of cooling, is considered by setting $A_{c} \leftarrow A_{c, \text { max }}$. This initially illustrates a setup like immersion cooling, see Fig. 8 . Next, the heat transfer coefficient $h$ is set to $h \leftarrow h_{f i x}$. This states a fixed material state while the temperature of the cooling fluid is considered to be adapted through iterations first. This treatment is based on choice and could be changed by fixing the fluid temperature first and iterating the heat transfer coefficient, when required. For $T_{f}$, an initial guess is made. Moreover a state indicator, denoting if the solution converged or not, is introduced and initially set to not converged state by state $\leftarrow 1$. A criterion for convergence of the solution is given by function as

$f\left(T_{j z}, T_{c}\right)=\left|T_{j z}-T_{c}\right|, \quad f\left(T_{j z}, T_{c}\right) \rightarrow \min$,

$f\left(T_{j z}, T_{c}\right)\left\{\begin{array}{l}\leq \text { tol converged } \\ \text { else not converged }\end{array}\right.$,

where $T_{j z}$ denotes the current temperature at the joining zone of the hybrid specimen and $T_{c}$ is a constraint, required for successful application of forming processes afterwards. The absolute value within Eq. (11) ensures that the cooling is not oversized. However, an acceptable temperature of the cooling fluid has to be considered by giving a tolerance $T_{f l} \geq$ tol $_{\text {temp }}$ which enables that within the iterative procedure the temperature is considered as fixed if a lower bound is reached once. For all states, where the temperature of the fluid is considered as the changing quantity, it is updated 
by an incremental change $\Delta T_{f l}$. Otherwise, the heat transfer coefficient will be updated within the iterative steps and the fluid temperature is held constant, respectively. The algorithm is summed up within Algorithm 1.

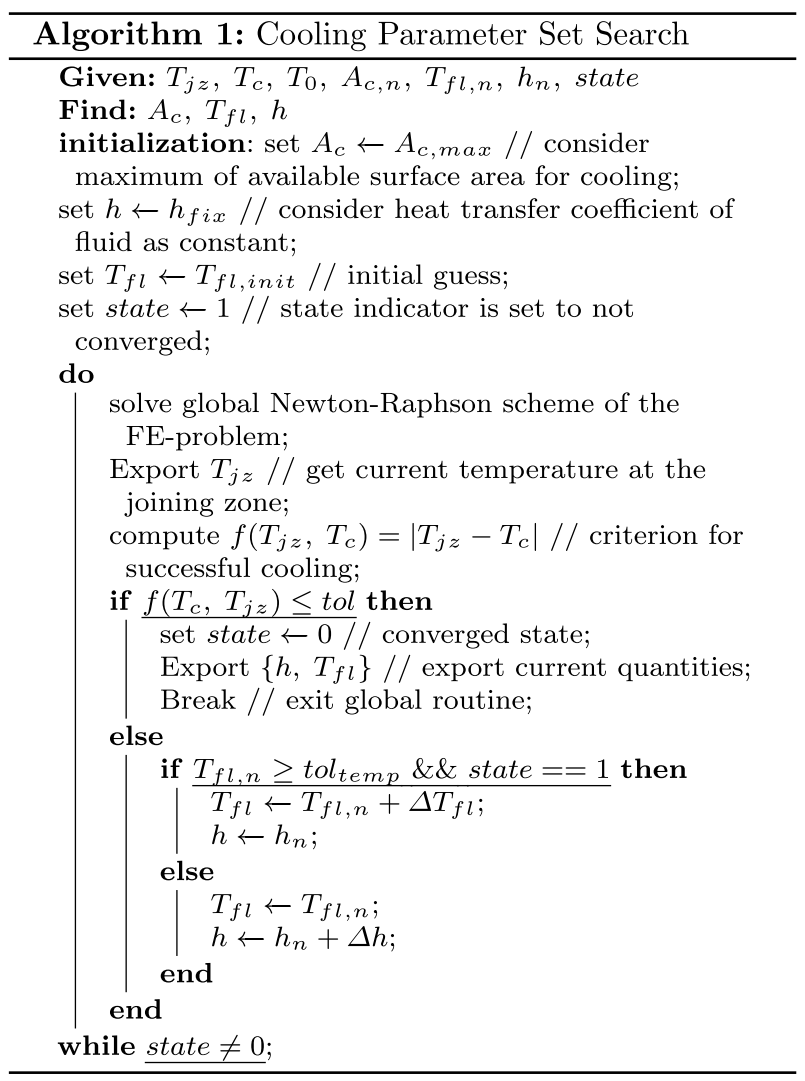

With that iterative procedure, a suitable set can be obtained by the computation of suitable ranges of the cooling-sensitive parameters $\left\{A_{c}, T_{f l}, h\right\}$. These quantities are needed within the design process for the optimisation of a cooling concept. This procedure can be used also to obtain an optimised surface area, to which a cooling is applied, by holding the subset $\left\{T_{f}, h\right\}$ constant, while solving $A_{c}\left(T_{f l}, h\right) \rightarrow \min$.

\section{Results}

First of all, the experimentally determined temperature curves that occur in the workpiece are presented. Then the cooling concept is presented, which is designed for the workpiece of the bearing bushing. The final part is a numerical simulation which shows the theoretical influence of the cooling on the heating strategy.

The temperature curves resulting from the heating tests on previously joined workpieces are shown in Fig. 7. The temperature increase after the intensive heating phase can

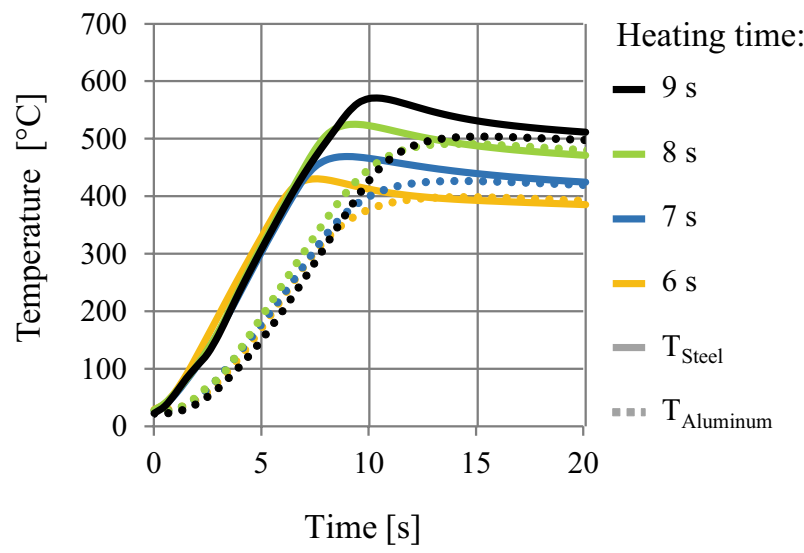

Fig. 7 Experimentally measured temperature curves within the mono-materials of a hybrid specimen for different time periods of induction heating

be explained by thermal lag of the thermocouples due to the relatively big diameter of their steel sheath. Slight deviation appears because of high heating rates during the intensive heating phase. The maximal heating time was restricted by the temperature equalisation at approx. $500{ }^{\circ} \mathrm{C}$ to avoid cracks in the aluminium part. The lower boundary for steel was defined in previous investigations, where a brittleness effect of steel was established at temperatures of around $450{ }^{\circ} \mathrm{C}[36]$. Because steel and aluminium are at equal temperature in the joining zone, the temperature of aluminium should be above this critical range. Only the strategy with $9 \mathrm{~s}$ heating and $2.5 \mathrm{~s}$ transfer time fulfils all requirements with the aluminium temperature of $480{ }^{\circ} \mathrm{C}$ and steel temperature of $570{ }^{\circ} \mathrm{C}$. By simultaneously cooling and heating in the ways described above, it is possible to increase the temperature of the steel without overheating the aluminium. Using this concept, higher temperature gradients, which were not taken into account before, can be used for forging.

As described above, the aim is to design a cooling system. For the initial boundary conditions spray cooling was already designed. In the context of the changed conditions, immersion cooling is to be also considered as useful. Immersion cooling offers the advantage of achieving a more homogeneous heat dissipation on the cooled surface than with spray cooling. The reason for this is that the fluid density decreases radially in the spray cone, whereby different heat flows occur. To generate locally different cooling rates and to achieve the desired temperature distribution in the workpiece with the immersion cooling the Leidenfrost effect shall be used. The cooling unit must be designed in such a way that the workpiece can be heated inductively. It must also be possible to position and remove the workpiece from the induction coil automatically. For handling, the gripping system presented by Ince et al. can still be used [26]. Therefore, the dimensions of the gripper must be taken into account when 
the container for the immersion cooling system is scaled. The coil must be physically separated from the fluid. The concept for implementing this immersion cooling is shown in Fig. 8.

The workpiece is placed on a ceramic tube which separates the induction coil from the fluid. There are inlets on each side of the tank. These serve to create a controlled flow in the tank, whereby an influence can be taken on the cooling rate. The liquid temperature can also be controlled by connecting the tank outlet with a heat exchanger, both are not illustrated in Fig. 8.

Nevertheless, it is planned that both processes, spray and immersion cooling, will be further elaborated.

Besides the development of a concept for immersion cooling, the development of a numerical scheme is an important objective, which enables the investigation for suitable parameter sets within the design process of the cooling. An algorithmic treatment of the model problem is illustrated and exhibits a general scheme which could also be utilised for the different geometric specimen in tailored forming processes, as well as for another type of cooling strategy. The effect of future changes in material or heating parameters on the required cooling rate can be easily determined by simulation.

Therefore, it is even more important that the simulation accurately represents the process of heating and the resulting temperature distribution. The simulation of the temperature distribution, described in Sect. 3.5, is depicted in Fig. 9 for a simultaneous heating and active cooling process, acting over a time-period of $11 \mathrm{~s}$. Parameters, used for the numerical study, are listed in Table 2. The input frequency of the current is $f=16300 \mathrm{~Hz}$. The electric field is accounted by its intensity as an input-quantity with $150 \frac{\mathrm{A}}{\mathrm{m}^{2}}$. The curves in Fig. 9 show a qualitative behavior of a possible immersion cooling setup. Since no experimental results are currently available for this

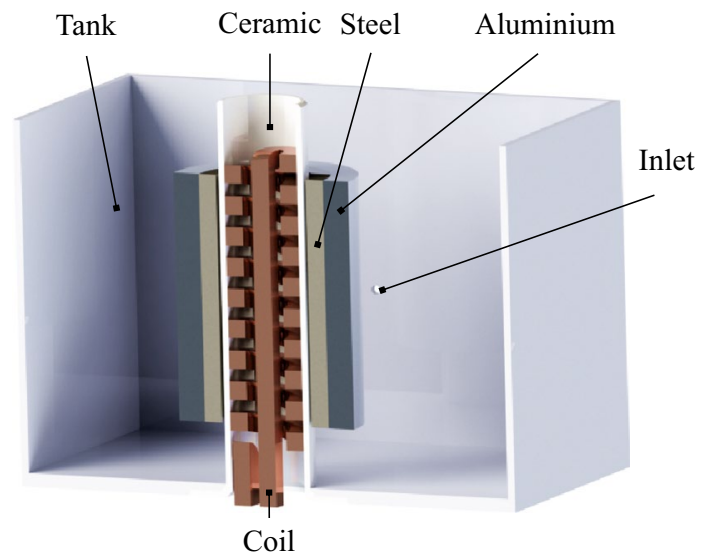

Fig. 8 Design concept of an immersion cooling system according to the boundary conditions including the workpiece of the bearing bushing and the induction coil
Table 2 Used parameters for the numerical study [37]

\begin{tabular}{|c|c|c|}
\hline Parameter & Steel & Aluminium \\
\hline Density $\rho\left[\frac{\mathrm{kg}}{\mathrm{m}^{3}}\right]$ & 7870 & 2700 \\
\hline Specific heat capacity $c\left[\frac{\mathrm{J}}{\mathrm{kgK}}\right]$ & 460 & 900 \\
\hline Thermal conductivity $k\left[\frac{\mathrm{w}}{\mathrm{mK}}\right]$ & 60 & 237 \\
\hline Electrical resistivity $\phi_{0}[\Omega \mathrm{m}]($ at $273.15 \mathrm{~K})$ & $1.43 \cdot 10^{-7}$ & $2.65 \cdot 10^{-8}$ \\
\hline Temperature coefficient $\alpha\left[\mathrm{K}^{-1}\right]$ & 0.004 & 0.004 \\
\hline Initial temperature $T_{0}[\mathrm{~K}]$ & 293.15 & 293.15 \\
\hline Permeability $\mu\left[\frac{\mathrm{H}}{\mathrm{m}}\right]$ & 100 & 1 \\
\hline
\end{tabular}

particular setup, the simulation has not been fitted to those specific setups yet.

The electric conductivity $\sigma$ is sensitive to the temperature $T$. In this setup the electrical resistivity $\phi$ is used, which is the inverse of the electric conductivity. Its evolution is captured by a linear dependency

$\phi=\phi_{0}\left[1+\alpha\left(T-T_{0}\right)\right], \quad \sigma=\phi^{-1}$.

The initial temperature of the cooling fluid is set to $T_{f, \text { init }}=273.15 \mathrm{~K}$ and the cooling rate is assumed to be $h=5 \cdot 10^{3} \frac{\mathrm{W}}{\mathrm{K} \mathrm{m}^{2}}$. It is obtained, that the induction heating has a high influence on the temperature, located within the aluminium material. Still, the cooling preserves a temperature at the joining zone $\left(\mathbf{X}_{J Z}\right)$, that fulfils the constraint $T_{J Z}\left(\mathbf{X}_{J Z}\right) \leq 500^{\circ} \mathrm{C}$. After $11 \mathrm{~s}$, the specimen is no longer under the influence of external heat sources. Thus, the temperature $T_{S t}\left(\mathbf{X}_{S t}\right)$, located within the steel material $\left(\mathbf{X}_{S t}\right)$, is undergoing a decrease while the temperature $T_{A l}\left(\mathbf{X}_{A l}\right)$ within the aluminium material $\left(\mathbf{X}_{A l}\right)$ is still increasing. This exchange develops towards an equilibrium state, which is located nearly $500{ }^{\circ} \mathrm{C}$. This simulation illustrates a possible

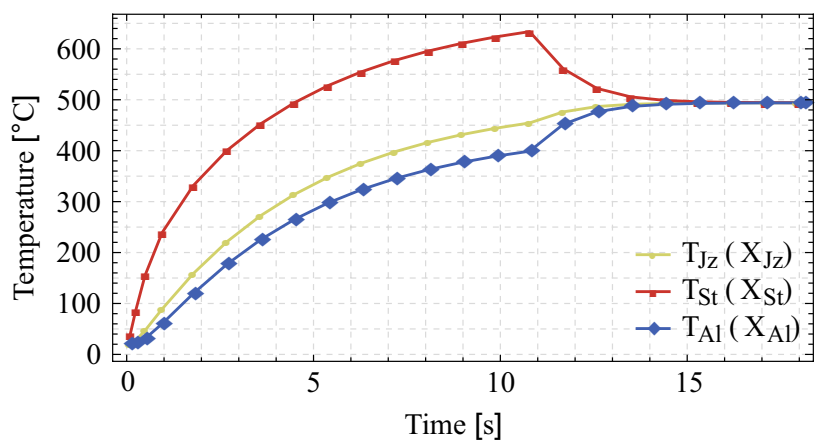

Fig. 9 Development of the temperatures $T_{i}\left(\mathbf{X}_{i}\right), i \in\{S t, J Z, A l\}$, located within the joining zone $(J Z)$, the steel $(S t)$ and the aluminium $(A l)$ material during a simultaneous active cooling and heating $t \in[0,11] s$ and their development afterwards 
treatment of heating and cooling as described before, by the immersion cooling setup, see Fig. 8. The result of the numerical investigation illustrates in general the applicability of longer heating times by considering an active cooling strategy, compare Fig. 7- Fig. 9.

\section{Conclusion}

The paper outlined a new concept for combining induction heating and cooling with the objective to improve the temperature range for the forging of steel-aluminium workpieces. The aim was to create an inhomogeneous heat distribution in the workpiece. Experimental heating tests showed that induction heating achieves such an inhomogeneous heat distribution. The tests also identified a parameter set which fulfils the material specific limitations sufficiently. Thereby the steel temperature is close to the lower boundary of the forming range.

Additional cooling of aluminium would help to increase the steel temperature without melting of the joining zone and achieving higher formability and better material properties. For this reason the manufacturing process of the bearing bushing was investigated in regard to a cooling strategy. The result of the investigation is that stationary cooling systems such as spray and immersion cooling are suitable for process integration.

To widen the range of parameter sets for multiple combinations of possible materials, a numerical scheme is presented. This scheme ensured an optimisation of cooling dependent parameters and was formulated in such a general way that no sensitivity towards specific geometries of the specimen or particular cooling concepts emerge. Thus, only material parameter dependencies are relevant, which allow the investigation of multiple heating/cooling approaches during a design process. The scheme showed that additional cooling has a positive effect on the achievable heating time. Compared to heating without cooling, the steel reaches a higher temperature, while the joining zone remains within a permissible temperature range. This strategy can be advantageous for more complex material combinations, such as aluminium and titanium, which will be investigated in future works.

\section{Outlook}

Future works will address the extension of thermal modelling to a highly coupled framework, i.e. as presented in [38, 39] or addressed in a virtual element framework in [29]. Furthermore, a development in the direction of a multiscale framework [40, 41] can also be considered. A further step is to implement the cooling strategy into the manufacturing process of the bearing bushing and thereby validate the model. This is the only way to ensure an accurate representation of the thermal behaviour of the hybrid components.

Acknowledgements The authors gratefully acknowledge the German research foundation (DFG, Deutsche Forschungsgemeinschaft) with the Collaborative Research Centre 1153 (CRC 1153) "Process chain for the production of hybrid high performance components through tailored forming" with the subprojects B02 Die forging of coaxially arranged hybrid workpieces, $\mathrm{C} 07$ Flexible handling of hot forging components and C04 Modelling of the joining zone, project number 252662854.

Funding Open Access funding enabled and organized by Projekt DEAL.

Open Access This article is licensed under a Creative Commons Attribution 4.0 International License, which permits use, sharing, adaptation, distribution and reproduction in any medium or format, as long as you give appropriate credit to the original author(s) and the source, provide a link to the Creative Commons licence, and indicate if changes were made. The images or other third party material in this article are included in the article's Creative Commons licence, unless indicated otherwise in a credit line to the material. If material is not included in the article's Creative Commons licence and your intended use is not permitted by statutory regulation or exceeds the permitted use, you will need to obtain permission directly from the copyright holder. To view a copy of this licence, visit http://creativecommons.org/licenses/by/4.0/.

\section{References}

1. Pervaiz M, Panthapulakkal S, KC B, Sain M, Tjong J (2016) Emerging trends in automotive lightweighting through novel composite materials. Materials Sciences and Applications 07(01):2638. https://doi.org/10.4236/msa.2016.71004

2. Martinsen K, Hu S, Carlson B (2015) Joining of dissimilar materials. CIRP Ann 64(2):679-699. https://doi.org/10.1016/j. cirp.2015.05.006

3. Wohletz S, Groche P (2014) Temperature influence on bond formation in multi-material joining by forging. Proc Eng 81:2000 2005. https://doi.org/10.1016/j.proeng.2014.10.271

4. Uhe J, Behrens B-A (2019) Manufacturing of hybrid solid components by tailored forming. In: Production at the leading edge of technology, pp 199-208. Springer Berlin Heidelberg. https://doi. org/10.1007/978-3-662-60417-5_20

5. Behrens B-A, Kosch K-G (2011) Development of the heating and forming strategy in compound forging of hybrid steel-aluminum parts. Materialwissenschaft und Werkstofftechnik 42(11):973978. https://doi.org/10.1002/mawe.201100795

6. Aldakheel F, Miehe C (2017) Coupled thermomechanical response of gradient plasticity. Int J Plasticity 91:1-24. https:// doi.org/10.1016/j.ijplas.2017.02.007

7. Aldakheel F (2016) Mechanics of nonlocal dissipative solids: Gradient plasticity and phase field modeling of ductile fracture. Ph.D. thesis, Institute of Applied Mechanics (CE), Chair I, University of Stuttgart. Http://dx.doi.org/10.18419/opus-8803

8. Aldakheel F (2017) Micromorphic approach for gradient-extended thermo-elastic-plastic solids in the logarithmic strain space. Continuum Mech Thermodyn 29(6):1207-1217

9. Aldakheel F, Hudobivnik B, Wriggers P (2019) Virtual elements for finite thermo-plasticity problems. Computat Mech 64(5):1347-1360 
10. Miehe C, Rosato D (2011) A rate-dependent incremental variational formulation of ferroelectricity. Int J Eng Sci 49(6):466-496

11. Zäh D, Miehe C (2013) Computational homogenization in dissipative electro-mechanics of functional materials. Comput Methods Appl Mech Eng 267:487-510

12. Miehe C, Welschinger F, Hofacker M (2010) A phase field model of electromechanical fracture. J Mech Phys Solids 58(10): 1716-1740

13. Guo X, Jin K, Wang H, Pei W, Ma F, Tao J, Kim N (2016) Numerical simulations and experiments on fabricating bend pipes by push bending with local induction-heating process. Int J Adv Manuf Technol 84(9-12):2689-2695

14. Song MC, Moon YH (2016) Coupled electromagnetic and thermal analysis of induction heating for the forging of marine crankshafts. Appl Thermal Eng 98:98-109. https://doi.org/10.1016/j. applthermaleng.2015.11.129

15. Goldstein R, Behrens B-A, Duran D (2017) Role of thermal processing in tailored forming technology for manufacturing multimaterial components. Heat treating, 29th Heat Treating Society Conference 29:172-179

16. Rudnev V, Loveless D, Cook R (2017) Handbook of induction heating. Manufacturing engineering and materials processing. CRC Press, Boca Raton

17. Kosch KG, Behrens B-A (2012) Challenges in compound forging of steel-aluminum parts. In: EPD Congress 2012, pp 169-176. Wiley Online Library

18. Chavdar B, Goldstein R, Yang X, Butkovich J, Ferguson L (2015) Hot hydroforging for lightweighting. In: 5th International conference on distortion engineering, Bremen, Germany

19. Mayinger F (1992) Thermo- and fluiddynamic principles of heat transfer during cooling. In: Theory and Technology of Quenching, pp 41-72. Springer Berlin Heidelberg. https://doi. org/10.1007/978-3-662-01596-4_3

20. Biance A-L, Clanet C, Quéré D (2003) Leidenfrost drops. Int J Heat Mass Transf 15(6):1632. https://doi.org/10.1063/1.1572161

21. Ramesh G, Prabhu NK (2011) Review of thermo-physical properties, wetting and heat transfer characteristics of nanofluids and their applicability in industrial quench heat treatment. Nanoscale Res Lett 6(1):334. https://doi.org/10.1186/1556-276X-6-334

22. Stephan P (2010) B1 fundamentals of heat transfer. In: VDI Heat Atlas, pp. 15-30. Springer Berlin Heidelberg, Berlin, Heidelberg. https://doi.org/10.1007/978-3-540-77877-6_115

23. Thürer SE, Peddinghaus J, Heimes N, Bayram FC, Bal B, Uhe J, Behrens B-A, Maier HJ, Klose C (2020) Lateral angular coextrusion: geometrical and mechanical properties of compound profiles. Metals 10(9):1162. https://doi.org/10.3390/met1009116 2

24. Hirschvogel M, Dommelen H (1992) Some applications of cold and warm forging. J Materials Process Technol 35(3-4):343-356. https://doi.org/10.1016/0924-0136(92)90326-n

25. Behrens B-A, Goldstein R, Chugreeva A (2018) Thermomechanical processing for creating bi-metal bearing bushings. Thermal Process Motion 2018:15-21

26. Ince C-V, Geggier J, Bruns C, Raatz A (2020) Development of a form-flexible handling technology with active cooling for hybrid components in forging processes. In: 8th CIRP conference of assembly technology and systems. In print
27. Jeschar R, Specht E, Köhler C (1992) Heat transfer during cooling of heated metallic objects with evaporating liquids. In: Liščić B, Tensi HM, Luty W (eds) Theory and technology of quenching: a handbook. Springer Berlin Heidelberg, Berlin, Heidelberg, pp 73-92. https://doi.org/10.1007/978-3-662-01596-4_4

28. Canale LdCF, Totten GE (2005) Quenching technology: a selected overview of the current state-of-the-art. Materials Res 8(4):461467. https://doi.org/10.1590/S1516-14392005000400018

29. Böhm C, Hudobivnik B, Marino M, Wriggers P (2020) Electromagneto-mechanically response of polycrystalline materials: Computational Homogenization via the Virtual Element Method. arXiv preprint arXiv:2008.01516

30. Zienkiewicz OC, Taylor RL, Nithiarasu P, Zhu J (1977) The finite element method, vol 3. McGraw-hill, London

31. Zohdi TI (2012) Electromagnetic properties of multiphase dielectrics: a primer on modeling, theory and computation. Springer Science \& Business Media, Berlin

32. Di Luozzo N, Fontana M, Arcondo B (2012) Modelling of induction heating of carbon steel tubes: mathematical analysis, numerical simulation and validation. J Alloys Compounds 536:S564-S568

33. Wheeler HA (1942) Formulas for the skin effect. Proc IRE 30(9):412-424

34. Hudobivnik B, Pajek L, Kunič R, Košir M (2016) Fem thermal performance analysis of multi-layer external walls during typical summer conditions considering high intensity passive cooling. Appl Energy 178:363-375

35. Korelc J, Wriggers P (2016) Automation of finite element methods. Springer International Publishing, Berlin. https://doi. org/10.1007/978-3-319-39005-5

36. Behrens B-A, Chugreev A, Matthias T (2018) Hybride Lagerbuchsen aus Aluminium und Stahl. Werkstatttechnik online 10(2018):691-697

37. MatWeb Material Property Data. http://www.matweb.com/index .aspx. Accessed: 2020-07-25

38. Schröder J, Keip MA (2010) A framework for the two-scale homogenization of electro-mechanically coupled boundary value problems. In: Computer methods in mechanics, pp 311-329. Springer

39. Aldakheel F (2011) Computational homogenization in microelectro-elasticity. Master's thesis. https://doi.org/10.13140/ RG.2.2.34176.66562

40. Schröder J, Lupascu DC (2017) Ferroic functional materials: experiment, modeling and simulation, vol 581. Springer, Berlin

41. Schröder J, Labusch M, Keip MA (2016) Algorithmic two-scale transition for magneto-electro-mechanically coupled problems: Fe2-scheme: localization and homogenization. Computer methods in applied mechanics and engineering 302:253-280

Publisher's Note Springer Nature remains neutral with regard to jurisdictional claims in published maps and institutional affiliations. 